\title{
Competencias Transversales: Una Revisión de las Mallas Curriculares de la Carrera de Odontología en Chile
}

\author{
Generic Competencies: A Review of Dental Curriculum Programs in Chile \\ Milena Soto Araya'; Josep E. Baños ${ }^{2}$; Jorge Pérez ${ }^{3}$ \& Elisabeth Moyano ${ }^{3}$
}

SOTO, A. M.; BAÑOS, J. E.; PÉREZ, J. \& MOYANO, E. Competencias transversales: una revisión de las mallas curriculares de la carrera de odontología en Chile. Int. J. Odontostomat., 15(4):928-937, 2021.

RESUMEN: El desarrollo de competencias transversales es un aspecto relevante en el currículum de pregrado de Odontología, sin embargo, existe escasa evidencia sobre su presencia en los planes de estudio nacionales. El objetivo de esta investigación fue conocer la presencia de asignaturas centradas en competencias transversales en la carrera de Odontología de las universidades chilenas. Se realizó un estudio descriptivo de diseño transversal, revisando las mallas curriculares publicadas en la web por cada universidad que dictó el pregrado de Odontología el año 2020, con proceso de admisión abierto. De las 19 universidades consultadas, todas exhibieron en sus mallas curriculares asignaturas asociadas a competencias transversales y distribuidas a lo largo de los seis años del plan de estudios. Al utilizar la agrupación de competencias transversales propuesta por el Proyecto Tuning América Latina, se observó que las asignaturas relacionadas con los procesos cognitivos asociados al aprendizaje eran las más numerosas y suponían el $41 \%$ del total; las vinculadas con los valores sociales correspondieron al $34 \%$; las asociadas al contexto tecnológico e internacional representaron el $20 \%$; mientras que las relacionadas con las habilidades interpersonales, solo alcanzaron un $5 \%$. Debido a su escasa presencia, es posible concluir que gran parte de los aprendizajes relacionados con el entrenamiento en habilidades interpersonales se encuentren resguardados en el currículum oculto y que probablemente estén a cargo del modelaje docente que se realiza diariamente en la práctica clínica. Como propuesta de futuro, el estudio sugiere mejorar la formación en competencias transversales de los egresados de odontología de las universidades chilenas.

PALABRAS CLAVE: competencias genéricas, competencias profesionales, educación superior, enseñanza en odontología, escuelas de odontología.

\section{INTRODUCCIÓN}

El currículum de la carrera de Odontología requiere conjugar una serie de conocimientos, habilidades, destrezas y actitudes en el proceso de enseñanza-aprendizaje de sus estudiantes. De acuerdo con la American Dental Education Association, el odontólogo general debiera ser educado para desarrollar, de manera independiente y autónoma, las siguientes competencias: profesionalismo, comportamiento ético, comunicación eficaz, habilidades interpersonales, evaluación y utilización de tecnologías emergentes, resolución de problemas, pensamiento crítico, así como también habilidades técnicas y procedimentales (ADEA, 2016).
Los planes de estudio de Odontología de las universidades chilenas dan cuenta de diferentes currículums, que representan interesantes propuestas de integración de ciencias básicas, preclínicas y clínicas, todo ello considerado como competencias específicas o "duras" que el odontólogo debe poseer. Las transversales, también conocidas como genéricas o "blandas", son comunes a cualquier titulación y necesarias para ejercer de manera eficaz una profesión. Sin embargo, no es frecuente que se consideren explícitamente en una asignatura determinada, al menos en ciencias de la salud (Palés-Argullós \& NollaDomenjó, 2016).

\footnotetext{
${ }^{1}$ Escuela de Odontología, Facultad de Salud y Odontología, Universidad Diego Portales, Santiago, Chile.

${ }^{2}$ Facultad de Medicina, Universitat de Vic - Universitat Central de Catalunya, Vic, España.

${ }^{3}$ Grupo de Investigación Educativa en Ciencias de la Salud (GRECS), Universidad Pompeu Fabra, Barcelona, España.
} 
Las competencias genéricas asumidas por cada carrera son las que han sido definidas previamente en el perfil académico-profesional y se incorporan al currículum para ser desarrolladas y evaluadas durante el período académico, con el fin de que los estudiantes las incorporen a su comportamiento (Villa \& Poblete, 2011). Se consideran imprescindibles para la formación de profesionales de cualquier campo del conocimiento; su desarrollo permite que los futuros egresados mejoren su nivel de empleabilidad y aprendan a gestionar su desarrollo personal y profesional (Alcota, 2009; Alcota et al., 2016; Rodríguez et al., 2019).

En Odontología, el currículum oculto o lo que los docentes transmiten a los estudiantes y que no se explicita en el currículum formal, cobra mayor relevancia en la práctica clínica, debido a que el profesor supervisor debiera actuar como modelo a seguir para los estudiantes, en relación a sus actitudes y comportamientos en la atención de pacientes (Alcota et al., 2012). En Chile, la docencia universitaria puede ser una segunda ocupación, por lo que el perfil docente es heterogéneo. En consecuencia, un importante número de docentes no poseen formación formal en pedagogía, lo que podría derivar en que muchos profesores se centren en el conocimiento específico disciplinar, más que en el desarrollo de competencias genéricas en el estudiantado (Villarroel \& Bruna, 2014).
El proyecto Tuning América Latina definió 27 competencias genéricas, a partir de la consulta realizada a estudiantes, académicos, egresados y empleadores de 19 países y 190 universidades latinoamericanas. Se agruparon en cuatro componentes principales o factores, atendiendo a la naturaleza de las competencias que representaban (Beneitone et al., 2007). La Tabla I presenta las competencias relacionadas con cada factor.

El objetivo de este estudio fue conocer la presencia de asignaturas centradas en competencias transversales, definidas en proyecto Tuning América Latina, en la carrera de Odontología de las universidades chilenas.

Si bien se ha remarcado la importancia de las competencias transversales (Alcota et al., 2016; Aravena et al., 2021; Fuentes \& Riquelme, 2021), no se ha estudiado la presencia de la formación en este tipo de competencias en Chile. Así, la presente investigación representa un punto de partida necesario para conocer el estado de la formación en valores profesionales y en competencias transversales en las carreras de Odontología de Chile con el fin de implementar acciones de mejora en este tipo de formación para los odontólogos generales chilenos.

Tabla I. Competencias genéricas agrupadas por factor según el Proyecto Tuning América Latina.

\begin{tabular}{ll}
\hline Grupo & Competencia genérica \\
\hline \multirow{3}{*}{ Factor 1: proceso de aprendizaje } & Capacidad de abstracción, análisis y síntesis \\
& Capacidad de aprender y actualizarse \\
& Conocimientos sobre el área de estudio y la profesión \\
& Capacidad para identificar, plantear y resolver problemas \\
& Capacidad crítica y autocrítica \\
& Capacidad de investigación \\
& Habilidades para buscar, procesar y analizar información \\
& Capacidad de comunicación oral y escrita \\
& Capacidad de aplicar los conocimientos en la práctica \\
\hline \multirow{3}{*}{ Factor 2: valores sociales } & Compromiso con su medio socio-cultural \\
& Valoración y respeto por la diversidad y multiculturalidad \\
& Responsabilidad social y compromiso ciudadano \\
& Compromiso con la preservación del medio ambiente \\
& Compromiso ético \\
\hline \multirow{3}{*}{ internacional } & Capacidad de comunicación en un segundo idioma \\
& Habilidad para trabajar en contextos internacionales \\
& Habilidades en el uso de las tecnologías de la información \\
\hline \multirow{2}{*}{ Factor 4: habilidades interpersonales } & Capacidad para tomar decisiones \\
& Habilidades interpersonales \\
& Capacidad de motivar y conducir hacia metas comunes \\
Capacidad de trabajo en equipo
\end{tabular}




\section{MATERIAL Y MÉTODO}

En base a la revisión de la información publicada en la web por cada universidad que dictó la carrera de Odontología el año 2020, se realizó un estudio descriptivo de corte transversal respecto de sus mallas curriculares.

En primera instancia, se buscó información oficial del Ministerio de Educación, que diera cuenta de las universidades que en el año 2020 impartieron la carrera de Odontología en Chile. Posteriormente, se revisaron las páginas web de todas las universidades que dictaron la carrera de Odontología, con el objetivo de conocer sus mallas curriculares (las webs consultadas son presentadas en la Tabla II). La indagación se concentró en la información más reciente publicada por cada universidad y relacionada con el proceso de admisión 2020.

A partir de la revisión de las mallas curriculares publicadas por cada universidad, se incluyeron en el estudio todas las asignaturas cuyos nombres aludieran a alguna competencia genérica y se excluyeron aquellos cursos cuyos nombres estuvieran relacionados con alguna competencia específica disciplinar. Adicionalmente, cada una de las competencias seleccionadas se asoció a uno de los cuatro factores, de acuerdo a la agrupación por análisis factorial propuesta por el proyecto Tuning América Latina (Beneitone et al.).

\section{RESULTADOS}

De acuerdo con lo informado por el Ministerio de Educación, las universidades que dictaron la carrera de Odontología en Chile con proceso de admisión abierto el año 2020 fueron 19 (Ministerio de Educación, 2020). Todas ofrecieron jornada única diurna, repartida en 11 o 12 semestres distribuidos a lo largo de los seis años del plan de estudios.

En la Tabla II se especifican las asignaturas relacionadas con las competencias transversales previstas en las mallas curriculares de todas las carreras de Odontología de las universidades chilenas. También se especifica el año y semestre donde se cursan y el factor del proyecto Tuning América Latina donde se ubican.

Las mallas curriculares de todas las universida- des consultadas contenían asignaturas asociadas al desarrollo de competencias transversales, las cuales se ofrecen repartidas a lo largo de todos los años del plan de estudios y están distribuidas en todos los semestres. Destaca también que 16 de las 19 universidades $(84,2 \%)$ ofrecen asignaturas explícitamente relacionadas con la formación en el método científico y la investigación; $15(78,9 \%)$ con la ética y la bioética; $14(73,6 \%)$ con el dominio de una lengua extranjera, mayoritariamente inglés; $10(52,6 \%)$ sobre bioestadística y también 10 (52,6\%) sobre algún aspecto relacionado con la comunicación. Como muestra la Tabla II, cada una de las asignaturas seleccionadas se asoció al factor de acuerdo con la agrupación por análisis factorial propuesta por el proyecto Tuning América Latina (Beneitone et al.). Algunas de las asignaturas recogidas en la Tabla II tienen un nombre genérico (Formación general, Electivo formación integral) que no ha permitido identificar qué competencias transversales concretas trabajan.

El resumen de cursos asociados al desarrollo de competencias transversales en las mallas curriculares de las universidades chilenas se muestra en la Tabla III. En ella se presenta el número de asignaturas agrupadas en cada una de las cuatro tipologías de competencias por universidad. Se registraron 194 asignaturas en total, con un promedio de 10 por universidad, con un rango entre 3 y 16 . Como se aprecia en la Figura 1, las materias más frecuentes estaban relacionadas con las competencias referentes a los procesos de aprendizaje (factor 1) representando el $40,7 \%$ del total. Las relacionadas con los valores sociales (factor 2) y con los contextos tecnológicos e internacionales (factor 3 ) representaron el $34 \%$ y el 20,1 $\%$ respectivamente. Las competencias sobre habilidades interpersonales (factor 4) fueron las menos frecuentes, representando únicamente el $5 \%$ del total.

\section{DISCUSIÓN}

Dada la relevancia de las competencias transversales en la formación del odontólogo general (Alcota et al., 2016; Aravena et al.; Fuentes \& Riquelme), nuestro objetivo consistió en determinar la presencia de dichas competencias en las carreras de Odontología de las universidades chilenas.

Los hallazgos de esta investigación muestran que, de las 19 universidades consultadas, todas exhiben en sus mallas curriculares cursos que se asocian 
SOTO, A. M.; BAÑOS, J. E.; PÉREZ, J. \& MOYANO, E. Competencias transversales: una revisión de las mallas curriculares de la carrera de odontología en Chile. Int. J. Odontostomat., 15(4):928-937, 2021.

Tabla II. Cursos asociados al desarrollo de competencias transversales en los planes de estudio de Odontología de universidades chilenas en el año 2020, año y semestre del plan de estudio en el que se imparte, y factor del proyecto Tuning América Latina en el que se incluye.

\begin{tabular}{|c|c|c|c|c|}
\hline Universidad & Año & Semestre & Curso & Factor \\
\hline \multirow{11}{*}{$\begin{array}{l}\text { Andrés Bello } \\
\text { https://www.unab.cl/carreras/mallas/odontologia.pdf }\end{array}$} & $1^{\circ}$ & $1^{\circ}$ & Formación General I & 2 \\
\hline & $1^{\circ}$ & $2^{\circ}$ & Inglés I & 3 \\
\hline & $2^{\circ}$ & $3^{\circ}$ & Inglés II & 3 \\
\hline & $2^{\circ}$ & $4^{\circ}$ & Formación General II & 2 \\
\hline & $2^{\circ}$ & $4^{\circ}$ & Inglés III & 3 \\
\hline & $3^{\circ}$ & $5^{\circ}$ & Inglés IV & 3 \\
\hline & $4^{\circ}$ & $7^{\circ}$ & Formación General III & 2 \\
\hline & $5^{\circ}$ & $9^{\circ}$ & Ética en la Práctica Odontológica & 2 \\
\hline & $5^{\circ}$ & $9^{\circ}$ & Metodología de la Investigación & 1 \\
\hline & $5^{\circ}$ & $10^{\circ}$ & Formación General IV & 2 \\
\hline & $6^{\circ}$ & $11^{\circ}$ y $12^{\circ}$ & P royecto Integrado de Investigación & 1 \\
\hline \multirow{8}{*}{$\begin{array}{l}\text { Arturo Prat } \\
\text { http://www.unap.cl/admision/2020/odontologia.html }\end{array}$} & $1^{\circ}$ & $1^{\circ}$ & E lectivo Formación General & 2 \\
\hline & $1^{\circ}$ & $2^{\circ}$ & Método Científico y Evidencia & 1 \\
\hline & $1^{\circ}$ & $2^{\circ}$ & E lectivo Forma ción General II & 2 \\
\hline & $2^{\circ}$ & $3^{\circ}$ & B ioestadística & 1 \\
\hline & $2^{\circ}$ & $3^{\circ}$ & E lectivo Forma ción General III & 2 \\
\hline & $4^{\circ}$ & $7^{\circ}$ & Bioética & 2 \\
\hline & $4^{\circ}$ & $8^{\circ}$ & Metodología de la Investigación & 1 \\
\hline & $6^{\circ}$ & $11^{\circ}$ y $12^{\circ}$ & Seminario de Tes is & 1 \\
\hline \multirow{9}{*}{$\begin{array}{l}\text { Austral } \\
\text { https://www.uach.cl/dw/admision/plandeestudio.php?car=1789 }\end{array}$} & $1^{\circ}$ & $1^{\circ}$ & Introduction to reading and listening strategies & 3 \\
\hline & $1^{\circ}$ & $2^{\circ}$ & Developing reading and listening strategies & 3 \\
\hline & $2^{\circ}$ & $3^{\circ}$ & OFG 1 & 2 \\
\hline & $2^{\circ}$ & $3^{\circ}$ & Unidad de integración. Ética y profesionalismo & 2 \\
\hline & $2^{\circ}$ & $3^{\circ}$ & In vestigación en Salud & 1 \\
\hline & $2^{\circ}$ & $4^{\circ}$ & OFG 2 & 2 \\
\hline & $5^{\circ}$ & $9^{\circ}$ & $\begin{array}{l}\text { Métodos Clínicos-Epidemiológicos de } \\
\text { In vestigación en Odontología }\end{array}$ & 1 \\
\hline & $5^{\circ}$ & $10^{\circ}$ & Proyecto de Tesina & 1 \\
\hline & $6^{\circ}$ & $11^{\circ}$ & Tesina & 1 \\
\hline \multirow{9}{*}{$\begin{array}{l}\text { Autónoma } \\
\text { https://cdn-ua.hostingreactor.com/ua admision/cache/wp- } \\
\text { content/uploads/2020/01/ODONTOLOGIA_MALLA_20 } \\
\text { 20.pdf }\end{array}$} & $1^{\circ}$ & $1^{\circ}$ & Electivo de Comunicación & 1 \\
\hline & $1^{\circ}$ & $2^{\circ}$ & Electivo de Comunicación & 1 \\
\hline & $2^{\circ}$ & $3^{\circ}$ & E lectivo de Desarrollo del Pensamiento & 1 \\
\hline & $2^{\circ}$ & $4^{\circ}$ & Electivo de Ética & 2 \\
\hline & $3^{\circ}$ & $5^{\circ}$ & B ioética & 2 \\
\hline & $3^{\circ}$ & $5^{\circ}$ & E lectivo de Responsabilidad Social & 2 \\
\hline & $3^{\circ}$ & $6^{\circ}$ & E lectivo de Desarrollo Personal & 4 \\
\hline & $5^{\circ}$ & $9^{\circ}$ & Metodología de la Investigación & 1 \\
\hline & $5^{\circ}$ & $10^{\circ}$ & P royecto de Investigación & 1 \\
\hline \multirow{11}{*}{$\begin{array}{l}\text { Católica de Chile } \\
\text { https://odontologia.uc.cl/pregrado/malla-curricular/ }\end{array}$} & $1^{\circ}$ & $1^{\circ}$ & B ioestadística & 1 \\
\hline & $1^{\circ}$ & $1^{\circ}$ & E lectivo Otra Disciplina Opt & 2 \\
\hline & $1^{\circ}$ & $1^{\circ}$ & Examen de Comunicación Escrita & 1 \\
\hline & $1^{\circ}$ & $1^{\circ}$ & Test de Inglés & 3 \\
\hline & $1^{\circ}$ & $2^{\circ}$ & E lectivo Otra Disciplina Opt & 2 \\
\hline & $2^{\circ}$ & $4^{\circ}$ & E lectivo Otra Disciplina Opt & 2 \\
\hline & $3^{\circ}$ & $5^{\circ}$ & E lectivo Otra Disciplina Opt & 2 \\
\hline & $3^{\circ}$ & $6^{\circ}$ & E lectivo Otra Disciplina Opt & 2 \\
\hline & $4^{\circ}$ & $7^{\circ}$ & Ética Médica & 2 \\
\hline & $4^{\circ}$ & $8^{\circ}$ & Seminario en Ciencias Odontológicas & 1 \\
\hline & $4^{\circ}$ & $8^{\circ}$ & E lectivo Otra Disciplina Opt & 2 \\
\hline \multirow{7}{*}{$\begin{array}{l}\text { De Antofagasta } \\
\text { http://odontologia.uantof.cl/carrera-de-odontologial }\end{array}$} & $1^{\circ}$ & $1^{\circ}$ & Inglés I & 3 \\
\hline & $1^{\circ}$ & $2^{\circ}$ & Desarrollo Personal e Interpersonal & 4 \\
\hline & $1^{\circ}$ & $2^{\circ}$ & Inglés II & 3 \\
\hline & $2^{\circ}$ & $3^{\circ}$ & Metodología de la Investigación Científica & 1 \\
\hline & $2^{\circ}$ & $4^{\circ}$ & Odontología Basada en la Evidencia I & 1 \\
\hline & $3^{\circ}$ & $5^{\circ}$ & Odontología Basada en la Evidencia II & 1 \\
\hline & $4^{\circ}$ & $7^{\circ}$ & B ioética & 2 \\
\hline
\end{tabular}


SOTO, A. M.; BAÑOS, J. E.; PÉREZ, J. \& MOYANO, E. Competencias transversales: una revisión de las mallas curriculares de la carrera de odontología en Chile. Int. J. Odontostomat., 15(4):928-937, 2021.

\begin{tabular}{|c|c|c|c|c|}
\hline Universidad & Año & Semestre & Curso & Factor \\
\hline & $4^{\circ}$ & $7^{\circ}$ & Electivo Formación Integral I & 2 \\
\hline & $4^{\circ}$ & $8^{\circ}$ & Electivo Formación Integral II & 2 \\
\hline & $6^{\circ}$ & $11^{\circ}$ & Electivo Formación Integral III & 2 \\
\hline \multirow{16}{*}{$\begin{array}{l}\text { Universidad de Chile } \\
\text { https://www.uchile.cl/carreras/5022/o dontologia }\end{array}$} & $1^{\circ}$ & $1^{\circ}$ & Sociedad, Cultura y Recreación & 2 \\
\hline & $1^{\circ}$ & $1^{\circ}$ & Inglés I & 3 \\
\hline & $1^{\circ}$ & $2^{\circ}$ & Infotecnologías e Instrumentos Informáticos & 3 \\
\hline & $1^{\circ}$ & $2^{\circ}$ & Sociedad, Cultura y Recreación & 2 \\
\hline & $1^{\circ}$ & $2^{\circ}$ & Inglés II & 3 \\
\hline & $2^{\circ}$ & $3^{\circ}$ & Odontología Basada en Evidencia I & 1 \\
\hline & $2^{\circ}$ & $3^{\circ}$ & Sociedad, Cultura y Recreación & 2 \\
\hline & $2^{\circ}$ & $3^{\circ}$ & Inglés III & 3 \\
\hline & $2^{\circ}$ & $4^{\circ}$ & Odontología Basada en Evidencia II & 1 \\
\hline & $2^{\circ}$ & $4^{\circ}$ & Sociedad, Cultura y Recreación & 2 \\
\hline & $2^{\circ}$ & $4^{\circ}$ & Inglés IV & 3 \\
\hline & $4^{\circ}$ & $7^{\circ}$ & Proyecto de Investigación I & 1 \\
\hline & $4^{\circ}$ & $8^{\circ}$ & Proyecto de Investigación II & 1 \\
\hline & $5^{\circ}$ & $9^{\circ}$ & Proyecto de Investigación III & 1 \\
\hline & $5^{\circ}$ & $10^{\circ}$ & Ejecución de Proyecto de Investigación & 1 \\
\hline & $6^{\circ}$ & $11^{\circ}$ y $12^{\circ}$ & Tesis de Grado & 1 \\
\hline \multirow{5}{*}{$\begin{array}{l}\text { De Concepción } \\
\text { https://admision.udec.cl/sites/default/files/sites/default/files/Odon } \\
\text { tologia.pdf }\end{array}$} & $1^{\circ}$ & $1^{\circ}$ & Inglés Comunicativo I Nivel Principiante & 3 \\
\hline & $1^{\circ}$ & $2^{\circ}$ & Inglés Comunicativo II Nivel Elemental & 3 \\
\hline & $2^{\circ}$ & $4^{\circ}$ & Metodología de la Investigación & 1 \\
\hline & $5^{\circ}$ & $9^{\circ}$ & Proyecto de Investigación & 1 \\
\hline & $5^{\circ}$ & $10^{\circ}$ & Proyecto de Investigación II & 1 \\
\hline \multirow{6}{*}{$\begin{array}{l}\text { De La Frontera } \\
\text { http://o dontologia.ufro.cl/index.php/carrera-de-odo ntologia }\end{array}$} & $1^{\circ}$ & $2^{\circ}$ & Idioma Inglés & 3 \\
\hline & $1^{\circ}$ & $2^{\circ}$ & Sociología y Desarrollo Comunitario & 2 \\
\hline & $3^{\circ}$ & $6^{\circ}$ & Electivo Cultural, Deportivo o Humanista & 2 \\
\hline & $4^{\circ}$ & $7^{\circ}$ & Bioética & 2 \\
\hline & $4^{\circ}$ & $7^{\circ}$ & Historia de la Cultura y Sociedad Chilena & 2 \\
\hline & $4^{\circ}$ & $8^{\circ}$ & Ética, Moral y Lógica & 2 \\
\hline \multirow{3}{*}{$\begin{array}{l}\text { De La Serena } \\
\text { http://www.admision.userena.cl/index.php/carreras/odontologia }\end{array}$} & $1^{\circ}$ & $2^{\circ}$ & Comunicación Oral y Escrita & 1 \\
\hline & $3^{\circ}$ & $5^{\circ}$ & Bioestadística & 1 \\
\hline & $3^{\circ}$ & $5^{\circ}$ & Metodología de la Investigación & 1 \\
\hline \multirow{12}{*}{$\begin{array}{l}\text { Del Desarrollo } \\
\text { https://medicina.udd.cl/odontologia-santiago/admision/malla- } \\
\text { curricular/ }\end{array}$} & $1^{\circ}$ & $1^{\circ}$ y $2^{\circ}$ & Electivo Track 1 y 2 Comunicación Escrita & 1 \\
\hline & $1^{\circ}$ & $1^{\circ}$ y $2^{\circ}$ & Electivo Track 1 y 2 Comunica ción Oral & 1 \\
\hline & $1^{\circ}$ & $1^{\circ}$ y $2^{\circ}$ & Electivo Track 1 y 2 Lectura $C$ rítica & 1 \\
\hline & $1^{\circ}$ & $1^{\circ}$ y $2^{\circ}$ & Electivo Track 1 y 2 Pensamiento Crítio & 1 \\
\hline & $1^{\circ}$ y $2^{\circ}$ & $1^{\circ}, 2^{\circ}$ y $3^{\circ}$ & $\begin{array}{l}\text { Créditos Extradisciplinares de Libre } \text { 日ección: Programa } \\
\text { Complementario de Inglés }\end{array}$ & 3 \\
\hline & $2^{\circ}$ & $3^{\circ}$ & Electivo Track 3 & 2 \\
\hline & $2^{\circ}$ & $4^{\circ}$ & Electivo Track 4 & 2 \\
\hline & $2^{\circ}$ & $4^{\circ}$ & Inglés Disciplinar & 3 \\
\hline & $4^{\circ}$ & $7^{\circ}$ & Epidemiologi a y Bioestadistica & 1 \\
\hline & $4^{\circ}$ & $7^{\circ}$ & Bioética & 2 \\
\hline & $4^{\circ}$ & $8^{\circ}$ & Investigación en Odontología I & 1 \\
\hline & $5^{\circ}$ & $9^{\circ}$ y $10^{\circ}$ & Investigación en Odontología II & 1 \\
\hline \multirow{10}{*}{$\begin{array}{l}\text { De Los Andes } \\
\text { https://admision.uandes.d/odontologia/ }\end{array}$} & $1^{\circ}$ & $2^{\circ}$ & Programa Optativo de Estudios Generales & 2 \\
\hline & $2^{\circ}$ & $3^{\circ}$ & Academia Científica & 1 \\
\hline & $2^{\circ}$ & $3^{\circ}$ & Bioestadística y Computación & 1 \\
\hline & $2^{\circ}$ & $3^{\circ}$ & Ética & 2 \\
\hline & $2^{\circ}$ & $3^{\circ}$ & Programa Optativo de Estudios Generales & 2 \\
\hline & $2^{\circ}$ & $4^{\circ}$ & Inglés & 3 \\
\hline & $3^{\circ}$ & $5^{\circ}$ & Metodología de la Investigación & 1 \\
\hline & $3^{\circ}$ & $6^{\circ}$ & Trabajo de Investigación & 1 \\
\hline & $3^{\circ}$ & $6^{\circ}$ & Programa Optativo de Estudios Generales & 2 \\
\hline & $6^{\circ}$ & $11^{\circ}$ & Ética Clínica & 2 \\
\hline
\end{tabular}


SOTO, A. M.; BAÑOS, J. E.; PÉREZ, J. \& MOYANO, E. Competencias transversales: una revisión de las mallas curriculares de la carrera de odontología en Chile. Int. J. Odontostomat., 15(4):928-937, 2021.

\begin{tabular}{|c|c|c|c|c|}
\hline Universidad & Año & Semestre & Curso & Factor \\
\hline & $6^{\circ}$ & $11^{\circ}$ & Preparación de Tesinas I & 1 \\
\hline & $6^{\circ}$ & $12^{\circ}$ & Preparación de Tesinas II & 1 \\
\hline \multirow{16}{*}{$\begin{array}{l}\text { De Talca } \\
\text { http://admision.utalca.cl/?page_id=2010 }\end{array}$} & $1^{\circ}$ & $1^{\circ}$ & Bioestadística Descriptiva & 1 \\
\hline & $1^{\circ}$ & $1^{\circ}$ & Idioma Extranjero I & 3 \\
\hline & $1^{\circ}$ & $1^{\circ}$ & Comunicación Oral y Escrita I & 1 \\
\hline & $1^{\circ}$ & $2^{\circ}$ & Idioma Extranjero II & 3 \\
\hline & $1^{\circ}$ & $2^{\circ}$ & Comunicación Oral y Escrita II & 1 \\
\hline & $2^{\circ}$ & $3^{\circ}$ & Idioma Extranjero III & 3 \\
\hline & $2^{\circ}$ & $3^{\circ}$ & Autogestión del Aprendizaje & 1 \\
\hline & $2^{\circ}$ & $4^{\circ}$ & Trabajo en Equipo y Desarrollo de Habilidades Sociales & 4 \\
\hline & $3^{\circ}$ & $5^{\circ}$ & Comprensión de Contextos Sociales & 2 \\
\hline & $3^{\circ}$ & $6^{\circ}$ & Comprensión de Contextos Culturales & 2 \\
\hline & $4^{\circ}$ & $7^{\circ}$ & Bioética & 2 \\
\hline & $4^{\circ}$ & $7^{\circ}$ & Ética y Responsabilidad Social & 2 \\
\hline & $4^{\circ}$ & $8^{\circ}$ & Responsabilidad Social & 2 \\
\hline & $6^{\circ}$ & $11^{\circ}$ & Proyecto de Memoria & 1 \\
\hline & $6^{\circ}$ & $11^{\circ}$ & Estadística Inferencial & 1 \\
\hline & $6^{\circ}$ & $12^{\circ}$ & Memoria & 1 \\
\hline \multirow{11}{*}{$\begin{array}{l}\text { De Valparaíso } \\
\text { https://odontologia.uv.cl/ }\end{array}$} & $1^{\circ}$ & $1^{\circ}$ & Taller de Autorregulación & 4 \\
\hline & $1^{\circ}$ & $2^{\circ}$ & Introducción a a Metodología Científica & 1 \\
\hline & $1^{\circ}$ & $2^{\circ}$ & Lengua Materna & 3 \\
\hline & $2^{\circ}$ & $3^{\circ}$ & Investigación en Ciencias de la Salud I & 1 \\
\hline & $2^{\circ}$ & $3^{\circ}$ & Inglés & 3 \\
\hline & $2^{\circ}$ & $4^{\circ}$ & OBE y Manejo de Información Científica & 1 \\
\hline & $3^{\circ}$ & $5^{\circ}$ & Investigación en Ciencias de la Salud II & 1 \\
\hline & $3^{\circ}$ & $6^{\circ}$ & Bioética Clínica & 2 \\
\hline & $5^{\circ}$ & $9^{\circ}$ & Investigación en Ciencias de la Salud III & 1 \\
\hline & $5^{\circ}$ & $10^{\circ}$ & Proyecto de Tesis & 1 \\
\hline & $6^{\circ}$ & $11^{\circ}$ & Proyecto y Difusión de Tesis & 1 \\
\hline \multirow{9}{*}{$\begin{array}{l}\text { Diego Portales } \\
\text { https://saludyodontologia. udp.clodonto logia/malla-curr icular/ }\end{array}$} & $1^{\circ}$ & $2^{\circ}$ & Curso de Formación General & 2 \\
\hline & $2^{\circ}$ & $3^{\circ}$ & Bioética & 2 \\
\hline & $2^{\circ}$ & $3^{\circ}$ & Curso de Formación General & 2 \\
\hline & $2^{\circ}$ & $4^{\circ}$ & Curso de Formación General & 2 \\
\hline & $4^{\circ}$ & $7^{\circ}$ & Curso de Formación General & 2 \\
\hline & $4^{\circ}$ & $8^{\circ}$ & Inglés General I & 3 \\
\hline & $5^{\circ}$ & $9^{\circ}$ & Inglés General II & 3 \\
\hline & $6^{\circ}$ & $11^{\circ}$ & Metodología de la Investigación & 1 \\
\hline & $6^{\circ}$ & $12^{\circ}$ & Seminario de la Investigación & 1 \\
\hline \multirow{15}{*}{$\begin{array}{l}\text { Finis Terrae } \\
\text { https://admision.uft.d/wp-content/uploads/2019/01/malla- } \\
\quad \text { odontologia.pdf }\end{array}$} & $1^{\circ}$ & $1^{\circ}$ y $2^{\circ}$ & Salud Pública e Investigación & 1 \\
\hline & $1^{\circ}$ & $1^{\circ}$ & Ser Universitario & 1 \\
\hline & $1^{\circ}$ & $1^{\circ}$ & Inglés I & 3 \\
\hline & $1^{\circ}$ & $2^{\circ}$ & Inglés II & 3 \\
\hline & $1^{\circ}$ & $2^{\circ}$ & Comunicación Efectiva & 4 \\
\hline & $2^{\circ}$ & $3^{\circ}$ y $4^{\circ}$ & Salud Pública e Investigación II & 1 \\
\hline & $2^{\circ}$ & $3^{\circ}$ & Inglés III & 3 \\
\hline & $2^{\circ}$ & $4^{\circ}$ & Electivo I & 2 \\
\hline & $2^{\circ}$ & $4^{\circ}$ & Inglés IV & 3 \\
\hline & $3^{\circ}$ & $5^{\circ}$ & Salud Pública e Investigación III & 1 \\
\hline & $3^{\circ}$ & $5^{\circ}$ & Ética & 2 \\
\hline & $3^{\circ}$ & $5^{\circ}$ & Liderazgo y Trabajo en Equipo & 4 \\
\hline & $3^{\circ}$ & $6^{\circ}$ & Electivo II & 2 \\
\hline & $3^{\circ}$ & $6^{\circ}$ & Creatividad y Resolución de Problemas & 1 \\
\hline & $6^{\circ}$ & $11^{\circ}$ y $12^{\circ}$ & Tesis de Grado & 1 \\
\hline
\end{tabular}


SOTO, A. M.; BAÑOS, J. E.; PÉREZ, J. \& MOYANO, E. Competencias transversales: una revisión de las mallas curriculares de la carrera de odontología en Chile. Int. J. Odontostomat., 15(4):928-937, 2021.

\begin{tabular}{|c|c|c|c|c|}
\hline Universidad & Año & Semestre & Curso & Factor \\
\hline \multirow{12}{*}{$\begin{array}{l}\text { Mayor } \\
\text { https://www.umayor.cl/um/bundles/carreras/descargables/fichas/ } \\
\text { odontologia.pdf }\end{array}$} & $1^{\circ}$ & $1^{\circ}$ & Competencias Académicas Universitarias & 1 \\
\hline & $1^{\circ}$ & $2^{\circ}$ & Escritura y Lectura Aca démica & 1 \\
\hline & $1^{\circ}$ & $2^{\circ}$ & Inglés I & 3 \\
\hline & $2^{\circ}$ & $3^{\circ}$ & Trabajo en Equipo & 4 \\
\hline & $2^{\circ}$ & $3^{\circ}$ & Comunicación Eficaz & 4 \\
\hline & $2^{\circ}$ & $3^{\circ}$ & Inglés II & 3 \\
\hline & $2^{\circ}$ & $4^{\circ}$ & Inglés III & 3 \\
\hline & $3^{\circ}$ & $5^{\circ}$ & Inglés IV & 3 \\
\hline & $4^{\circ}$ & $7^{\circ}$ & Elaboración y Evaluación de Proyectos & 1 \\
\hline & $5^{\circ}$ & $9^{\circ}$ & Bioestadística y Metodología de la Investigación & 1 \\
\hline & $5^{\circ}$ & $10^{\circ}$ & Taller de Emprendimiento & 4 \\
\hline & $6^{\circ}$ & $11^{\circ}$ y $12^{\circ}$ & Trabajo de Investigación & 1 \\
\hline \multirow{4}{*}{$\begin{array}{l}\text { San Sebastián } \\
\text { https://www.uss.cl/odontologia/carrera/odontologia/malla- } \\
\text { curricular/ }\end{array}$} & $2^{\circ}$ & $3^{\circ}$ & Bioética & 2 \\
\hline & $2^{\circ}$ & $3^{\circ}$ & Formación Integral I & 2 \\
\hline & $2^{\circ}$ & $4^{\circ}$ & Formación Integral II & 2 \\
\hline & $3^{\circ}$ & $5^{\circ}$ & Formación Integral III & 2 \\
\hline \multirow{11}{*}{$\begin{array}{l}\text { Viña del Mar } \\
\text { https://www.uvm.cl/escuela-de-ciencias-de-la-salud/odontologia/ }\end{array}$} & $1^{\circ}$ & $1^{\circ}$ & Gestión Personal & 4 \\
\hline & $1^{\circ}$ & $2^{\circ}$ & Expresión Oral y Escrita & 1 \\
\hline & $1^{\circ}$ & $2^{\circ}$ & Herramientas y Métodos Básicos para el Trabajo Científico & 1 \\
\hline & $2^{\circ}$ & $3^{\circ}$ & Epidemiología y Bioestadística & 1 \\
\hline & $2^{\circ}$ & $3^{\circ}$ & Formación General & 2 \\
\hline & $2^{\circ}$ & $3^{\circ}$ & Inglés I & 3 \\
\hline & $2^{\circ}$ & $4^{\circ}$ & Inglés II & 3 \\
\hline & $3^{\circ}$ & $5^{\circ}$ & Inglés III & 3 \\
\hline & $3^{\circ}$ & $6^{\circ}$ & Inglés IV & 3 \\
\hline & $4^{\circ}$ & $8^{\circ}$ & Formación General & 2 \\
\hline & $5^{\circ}$ & $9^{\circ}$ & Método Científico y Proyecto en Salud & 1 \\
\hline
\end{tabular}

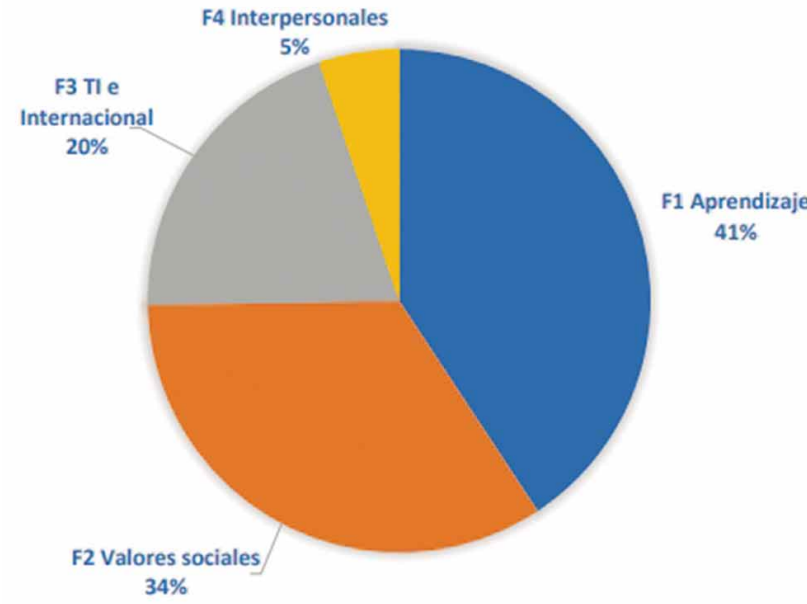

Fig. 1. Distribución porcentual de las asignaturas asociadas a competencias transversales en la carrera de Odontología en universidades chilenas con proceso de admisión abierto el año 2020, agrupadas según los factor del proyecto Tuning América Latina. F1; factor 1; F2: factor 2; F3: Factor 3; TI: tecnología de las información; F4: factor 4. a competencias transversales. Cada institución posee, en promedio, 10 asignaturas de esta naturaleza y éstas se encuentran distribuidas a lo largo de los seis años del plan de estudios.

Al agrupar las competencias transversales en factores, se hace evidente que el relacionado con el factor 1 es el que concentra la mayor parte de asignaturas, correspondiendo al $41 \%$ del total de cursos asociados a competencias transversales, con un promedio de 4,16 por institución. Este factor está relacionado con aquellas competencias transversales que aluden a los procesos cognitivos asociados al aprendizaje, por lo que resulta razonable que las instituciones de educación superior las consideren de gran trascendencia. Recordemos que asignaturas relacionadas con la investigación, el método científico o con la bioestadística se ofertaban en la mayoría de las universidades. 
Tabla III. Asignaturas asociadas a competencias transversales presentes en la malla curricular de la carrera de Odontología en universidades chilenas con proceso de admisión abierto el año 2020, agrupadas según factor de Tuning.

\begin{tabular}{|c|c|c|c|c|c|}
\hline UNIVERSIDAD & FACTOR 1 & FACTOR 2 & FACTOR 3 & FACTOR 4 & TOTAL \\
\hline Andrés Bello & 2 & 5 & 4 & 0 & 11 \\
\hline Arturo Prat & 4 & 4 & 0 & 0 & 8 \\
\hline Austral de Chile & 4 & 3 & 2 & 0 & 9 \\
\hline Autónoma de Chile & 5 & 3 & 0 & 1 & 9 \\
\hline Católica de Chile & 3 & 7 & 1 & 0 & 11 \\
\hline De Antofagasta & 3 & 4 & 2 & 1 & 10 \\
\hline De Chile & 7 & 4 & 5 & 0 & 16 \\
\hline De Concepción & 3 & 0 & 2 & 0 & 5 \\
\hline De La Frontera & 0 & 5 & 1 & 0 & 6 \\
\hline De La Serena & 3 & 0 & 0 & 0 & 3 \\
\hline Del Desarrollo & 7 & 3 & 2 & 0 & 12 \\
\hline De Los Andes & 6 & 5 & 1 & 0 & 12 \\
\hline De Talca & 7 & 5 & 3 & 1 & 16 \\
\hline De Valparaíso & 7 & 1 & 2 & 1 & 11 \\
\hline Diego Portales & 2 & 5 & 2 & 0 & 9 \\
\hline Finis Terrae & 6 & 3 & 4 & 2 & 15 \\
\hline Mayor & 5 & 1 & 4 & 3 & 13 \\
\hline San Sebastián & 1 & 4 & 0 & 0 & 5 \\
\hline Viña del Mar & 4 & 4 & 4 & 1 & 13 \\
\hline Total & 79 & 66 & 39 & 10 & 194 \\
\hline Promed io & 4,1 & 3,4 & 2,0 & 0,5 & 10,2 \\
\hline
\end{tabular}

Las competencias transversales del factor 2 son aquellas ligadas con los valores sociales, y que son del todo relevantes en los profesionales de la salud, quienes asumen un rol de servicio con la comunidad. Es así como el $34 \%$ de los cursos asociados a competencias transversales son de esta naturaleza, con un promedio de 3,47 por universidad. Ello es debido a que la formación referente al comportamiento ético de los profesionales está presente en la mayoría de las universidades.

Al revisar las competencias genéricas asociadas al factor 3 , estas corresponden al $20 \%$ del total de cursos presentados, con un promedio de 2 por institución. En este grupo se ubican las asociadas al contexto tecnológico e internacional, que en la presente investigación se relacionan fundamentalmente con el dominio del idioma inglés, que también es ofertado en la gran mayoría de las universidades.

Finalmente, las competencias del factor 4 corresponden a las habilidades interpersonales, cuyo manejo es vital para el desarrollo de una óptima relación odontólogo-paciente, así como también en las relaciones que el odontólogo establece con sus pares y los otros profesionales sanitarios. En esta categoría encontramos que solo un $5 \%$ de los cursos de com- petencias transversales obedecen a esta naturaleza, exhibiendo un promedio de 0,5 por universidad.

En Chile, se estudió la composición del currículum de las escuelas de pregrado de Odontología entre los años 2010 al 2014. Sus autores concluyeron que las ciencias clínicas son el grupo de cursos más dominante en el currículum dental chileno (33\%), seguido por ciencias básicas y biológicas (16\%) y luego por ciencias médicas y dentales (13\%), estableciendo así que el currículum se ha enfocado en el ámbito de la Odontología restauradora de manera desproporcionada. Cabe mencionar que en este estudio se trabajó con nueve categorías de cursos, ninguno de los cuales aludía explícitamente a competencias transversales (Venturelli Garay \& Watt, 2018).En este sentido, se ha señalado que en la mayoría de los currículos de Odontología se observa una carencia de horario protegido destinado al desarrollo de competencias transversales, a diferencia de lo que sucede en países con reconocidos estándares de calidad en la formación profesional de odontólogos (Alcota et al., 2016).

En el pregrado de Odontología de la Universidad de La Frontera de Chile se llevó a cabo una renovación curricular, en la que uno de sus tres ejes centrales fue el desarrollo de competencias genéricas, en- 
tendiéndolo como una "herramienta indispensable en el mundo actual para enfrentar con acierto los cambios y desafíos de una sociedad que demanda cada vez más la formación integral del ser humano" (Fuentes \& Riquelme). En este proceso se abordaron los desafíos de la educación en Odontología, tales como el predominio de un enfoque clínico individualista y la discordancia entre la educación dental, los programas de internado y las necesidades en salud oral de la comunidad local. Para esto, en su currículum se realzó la educación profesional interdisciplinaria y holística a través del desarrollo de las siguientes competencias genéricas:comunicación oral y escrita, aprender a aprender, pensamiento complejo, liderazgo, trabajo en equipo y responsabilidad social (Aravena et al.).

En Reino Unido se ha estudiado el rol de las ciencias sociales y del comportamiento en el currículum de pregrado de Odontología (Neville \& Waylen, 2019). Se indicó que, para muchos estudiantes, es difícil reconocer su importancia, pues prefieren los aspectos técnicos de la carrera sobre los sociales. En este sentido, se observó una ambivalencia hacia las ciencias sociales y del comportamiento, atribuido a dos causas: la hegemonía del enfoque biomédico y el currículum oculto. El primero se refiere al énfasis de la habilidad técnica y del manejo clínico de las enfermedades orales, predominando un enfoque individualista que no entrega a los estudiantes herramientas suficientes para lograr cambios sostenibles al largo en plazo en salud oral. El currículum oculto, por su parte, se refiere a la experiencia vivida por los estudiantes y que considera la influencia de docentes y pares, así como también los valores y juicios morales de la profesión. En educación médica, el currículum oculto está asociado con el desarrollo del pensamiento ético, la empatía y el idealismo (Palés-Argullós, 2015), pero en Odontología hay menos investigación al respecto. Los aspectos ya mencionados perpetúan la idea equivocada que la Odontología es una disciplina puramente clínica y no una disciplina biopsicosocial y ponen en peligro el foco que debiera tener al servicio del paciente. De aquí la necesidad de desarrollar en el estudiante de pregrado habilidades de comunicación y de manejo del paciente (Neville \& Waylen).

Llama la atención los pocos cursos que a nivel nacional fueron diseñados para desarrollar habilidades interpersonales en los estudiantes de Odontología. En consecuencia, es posible considerar que gran parte de estos aprendizajes se encuentren en el currículum oculto, a cargo del modelaje docente que se realiza diariamente en la práctica clínica. En este con- texto, parece necesario prestar especial atención a los perfiles docentes de los profesionales que se contratan para estos fines, y en cuyas manos se encuentra la mayor parte del aprendizaje de los estudiantes en habilidades interpersonales. También se requeriría reevaluar los planes de estudio, con una mirada menos disciplinar y más cercana a las competencias que tanto valoran los empleadores y que son fundamentales para una práctica responsable del odontólogo general y la consolidación de una óptima relación odontólogo-paciente.

En nuestra revisión hemos encontrado artículos relevantes sobre la formación universitaria de los futuros odontólogos chilenos (Alcota et al., 2016; Lagos et al., 2018; Venturelli Garay \& Watt; Aravena et al.; Fuentes \& Riquelme). En alguno de ellos se resaltaba la importancia de la formación en competencias genéricas (Alcota, et al., 2016; Aravena et al.; Fuentes \& Riquelme), pero no tenemos conocimiento de estudios sobre la situación de dicha formación en las carreras de Odontología de las universidades chilenas. Por ello consideramos que el presente estudio permite visualizar de manera preliminar el estado de las competencias transversales en la formación de odontólogos generales en Chile.

Si el propósito es seleccionar la intervención más adecuada para superar las debilidades de cada universidad en este ámbito, nuestro estudio se puede complementar con la información proveniente de los planes de estudio y programas académicos de cada institución específicamente. Consideramos que la sola revisión de las mallas curriculares puede tener limitaciones y hacer un análisis concreto puede ayudar a identificar las debilidades en esta área.

Podemos concluir que la información aportada por el presente estudio permite esbozar acciones de mejora para fomentar la formación en competencias genéricas de los egresados de las carreras de Odontología de las universidades chilenas, así como también relevar la necesidad de formación en el ámbito de la pedagogía universitaria delos equipos docentes, que permita asegurar un óptimo desempeño de su rol.

SOTO, A. M.; BAÑOS, J. E.; PÉREZ, J. \& MOYANO, E. Generic competencies: a review of dental curriculum programs in Chile. Int. J. Odontostomat., 15(4):928-937, 2021.

ABSTRACT: The development of transversal competencies is a relevant aspect in the dental undergraduate curriculum. However, there is insufficient 
evidence about its implementation in national curriculums. The aim of this research was to know whether or not the courses are focused on transversal competencies in dental programs of Chilean universities. To accomplish this, a descriptive, cross-sectional study was carried out. This research was based on the review of the curriculums published on the websites of each university that awarded undergraduate degrees in dentistry in 2020, and facilitated an open admission process. Of the 19 universities studied, all had courses associated with transversal competencies in their dental curriculum. These were distributed throughout the six years of the curriculum. Using the grouping of transversal competencies, as proposed by the Latin America Tuning Project, it was shown that courses related to cognitive learning processes were the most represented of the subjects, corresponding to $41 \%$ of all courses studied. Those related to social values corresponded to $34 \%$; courses associated with informational technology and those within the international context represented $20 \%$; while the courses related to interpersonal skills only reached $5 \%$. Due to the fact that the courses related to interpersonal skills were scarce, it could be concluded that a large part of this learning is included in the hidden curriculum and is probably achieved via instructional modeling as part of the daily clinical practice. As a proposal for the future, this research suggests that we need to improve the training in transversal competencies for the dental graduates of Chilean universities.

KEY WORDS: generic skills, professional competencies, higher education, dental education, dental schools

\section{REFERENCIAS BIBLIOGRÁFICAS}

ADEA. Competencies for the New General Dentist. J. Dent. Educ., 80(7):825-8, 2016.

Alcota, M.; Ruiz de Gauna, P. \& González, F. E. Dental programs in the current context of Chilean higher education. Int. J. Odontostomat., 10(1):85-91, 2016.

Alcota, M.; Ruiz de Gauna, P. \& González, F. E. Development of ethical practices and social responsability in dental education at the university of Chile: student and faculty perceptions. Eur. J. Dent. Educ., 17(1):70-6, 2013.

Alcota, M.P. Aportes de la Carrera de Odontología al Desarrollo de Competencias Genéricas: Compromiso Ético y Responsabilidad Social. Tesis para la obtención del Grado de Magister en Educación en Ciencias de la Salud. Santiago de Chile, Facultad de Medicina, Universidad de Chile, 2009.

Aravena, R. Y.; Huanquilef, A. M.; Arellano, V. M.; Nuñez, C. J. \& Fuentes, F. R. A report of the interdisciplinary education approach within the Universidad de La Frontera Dental Curriculum. Int. J. Odontostomat., 15(1):300-5, 2021.

Beneitone, P.; Esquetini, C.; González, J.; Marty Maletá, M.; Siufi, G. \& Wagenaar, R. (Eds.). Reflexiones y Perspectivas de la Educación Superior en América Latina. Informe Final - Proyecto Tuning - América Latina 2004-2007. Universidad de Deusto, Universidad de Groningen. Bilbao, Publicaciones de la Universidad de Deusto, 2007. pp.33-70.
Alcota, M.; Ruiz de Gauna, P. \& González, F. Development of ethical practices and social responsibility in dental education at the university of Chile: student and faculty perceptions. Eur. J. Dent. Educ., 17(1):e70-e76, 2012

Fuentes, F. R. \& Riquelme, M. C. Background and considerations for the curricular renewal of the Universidad de La Frontera, Chile dental degree. Int. J. Odontostomat., 15(1):234-9, 2021.

Lagos, T. D.; Bravo, R. L.; Ramos, M. J. L. \& Ciocca, G. L. Teaching and research in legal dentistry in Chile: current situation and challenges. Int. J. Odontostomat., 12(3):274-9, 2018.

Ministerio de Educación (MINEDUC). Mi Futuro. Carrera Odontología. Santiago de Chile, Ministerio de Educación, Gobierno de Chile, 2020. Disponible en: https://www.mifuturo.cl/buscador-decarreras $/$ ?tipo $=$ carrera\&find $=$ crr\&area $=9 \&$ titulo $=953 \&$ pagina $=1$

Neville, P. \& Waylen, A. Why UK dental education should take a greater interest in the behavioural and social sciences. Br. Dent. J., 227(8):667-70, 2019.

Palés-Argullós, J. \& Nolla-Domenjó, M. Competencias transversales, un tema pendiente en las facultades de medicina. FEM, 19(5):227, 2016.

Palés-Argullós, J. Estrategias para el aprendizaje del profesionalismo. Educ. Med., 16(Supl. 1):13-6, 2015.

Rodríguez, A.; Cortés M. \& Val, S. Análisis de la mejora del nivel de empleabilidad de los universitarios mediante la mejora de competencias transversales y habilidades. REOP, 30(3):102-19, 2019.

Venturelli Garay, R. E. \& Watt, R. G. Review and analysis of Chilean dental undergraduate education: Curriculum composition and profiles of first year dental students. Hum. Resour. Health, 16(1):1-9, 2018.

Villa, A. \& Poblete, M. Assessment of generic competencies: Principles, opportunities and limitations. Bordon, 63(1):147-70, 2011.

Villarroel, V. \& Bruna, D. Reflexiones en torno a las competencias genéricas en educación superior: Un desafío pendiente. Psicoperspectivas, 13(1):23-34, 2014.

Dirección para correspondencia:

Dra. Milena Soto Araya

Escuela de Odontología

Facultad de Salud y Odontología

Universidad Diego Portales

Santiago

CHILE

E-mail: milena.soto@udp.cl 